\title{
Dynamic Clustering for Vigilance Analysis Based on EEG
}

\author{
Li-Chen Shi and Bao-Liang Lu* Senior Member, IEEE
}

\begin{abstract}
Electroencephalogram (EEG) is the most commonly studied signal for vigilance estimation. Up to now, many researches mainly focus on using supervised learning methods for analyzing EEG data. However, it is hard to obtain enough labeled EEG data to cover the whole vigilance states, and sometimes the labeled EEG data may be not reliable in practice. In this paper, we propose a dynamic clustering method based on EEG to estimate vigilance states. This method uses temporal series information to supervise EEG data clustering. Experimental results show that our method can correctly discriminate between the wakefulness and the sleepiness for every 2 seconds through EEG, and can also distinguish two other middle states between wakefulness and sleepiness.
\end{abstract}

\section{INTRODUCTION}

Vigilance, or sustained attention, refers to the ability of observers to maintain their focus of attention and remain alert to stimuli for a prolonged period of time. During the past few decades, studies on vigilance have shown that vigilance estimation is very useful to our daily lives [1] [2]. In the past few decades, various signals were used to analyze vigilance. Among them, electroencephalogram (EEG) is the most commonly studied signal for vigilance estimation . In EEG-based vigilance research, several important phenomenon have been discovered [3]. These include decreased amplitude of event-related potentials (ERPs) with decreasing vigilance, increased theta activity with decreasing vigilance, and decreased beta activity with decreasing vigilance. Most existing methods have focused on employing supervised learning methods to estimate vigilance states [4] [5] [6] [7].

However, till now, there is no standard criteria for vigilance scale labeling, and the existing vigilance labeling methods are complex, expensive and sometimes unreliable. Considering these situations, we choose clustering methods to mine the latent distribution of EEG for vigilance estimation [8]. Furthermore, many studies show that, the vigilance state transition during a long period is a gradually changing process [2] [7] [3]. Therefore, we can use the time series relations among EEG signals to design the clustering algorithm. Because the labeled EEG data of wakefulness and sleepiness are easy to be obtained, we can use these labeled information to supervise the EEG spatial filtering process. Comparing to other traditional clustering methods such as k-means and normalized cuts (ncuts) algorithm [9], our proposed clustering method has the following two main

Asterisk indicates corresponding author. This work was supported in part by the National Natural Science Foundation of China under the grants NSFC 60473040 and NSFC 60773090, the Microsoft Laboratory for Intelligent Computing and Intelligent Systems of Shanghai Jiao Tong University, and the Okawa Foundation Research Grant. L. C. Shi and B. L. Lu are with the Department of Computer Science and Engineering, Shanghai Jiao Tong University, Shanghai 200240 China. E-mail: \{lch-shi; bllu $\} @$ sjtu.edu.cn. advantages. a) It not only makes use of current observation of EEG signals but also nearby observations to determine the current vigilance state. b) The ultimate vigilance state sequence is regarded as a globally optimal solution for all the observations. This is just consistent with the actual fact that vigilance is a gradually changing process and states are dependant on each other.

This paper is organized as follows. In Section II, vigilance experimental setup is introduced. In Section III, data analysis process and the proposed dynamic clustering algorithm are presented. In Section IV, experimental results are described. Finally, some conclusions are given in Section V.

\section{Methodology}

In this study, totally 17 healthy subjects of 19 to 35 years old have participated in our experiment. They are not allowed to take alcohol or caffeine within one day before the experiment. Each subject has finished at least four sessions, which are carried out in a small sound proof room with normal illumination. Each session lasts for about one hour, from 13:00 to 14:00 after lunch.

During the experiment, the subjects with earphones are required to lie on a bed, close their eyes, and try to relax. During this time, a short period of soft music is presented to the subject about every 10 minutes. The music lasts 10 seconds with the volume that does not disturb the sleeping subjects. The subjects are required to open their eyes to demonstrate they are awake if they hear the music.

The who process is recorded by a digital video camera. Meanwhile, a total number of 64 channels of signals including 62 channels of EEG and 2 channels of EOG were recorded through the NeuroScan system sampled at $1000 \mathrm{~Hz}$, and are filtered between 0.1 and $100 \mathrm{~Hz}$. The electrodes are arranged based on extended 10/20 system. Reference points are put on the papillary place behind two ears.

After each experiment, we use the feedback information from the subject and combine with the video recording to manually label the wakefulness state and sleepiness state of each subject. The EEG data were labeled, only when both sides had the same vigilance state assessment.

\section{Data Analysis}

We use multi-channels EEG signals for vigilance estimation. The whole process consists of six parts. The flow chart of data analysis for vigilance estimation is shown in Fig. 1.

\section{A. Noise and Artifacts Removal}

In the experiment, we firstly use a band-pass filter $(1 \mathrm{~Hz}-$ $50 \mathrm{~Hz})$ to remove the low-frequency noise and the highfrequency noise. Then, we use independent components 


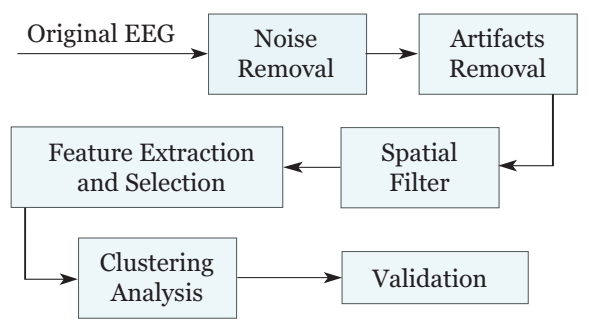

Fig. 1. Flow chart of data analysis for vigilance estimation.

analysis (ICA) [10] to remove the artifacts. After calculating the spectrum of each independent component, we found that the spectrum of artifact is much more different than the average spectrum, as shown in Fig. 2. As a result, we can remove the artifacts and reconstruct the EEG signals based on the spectrum of each independent component.

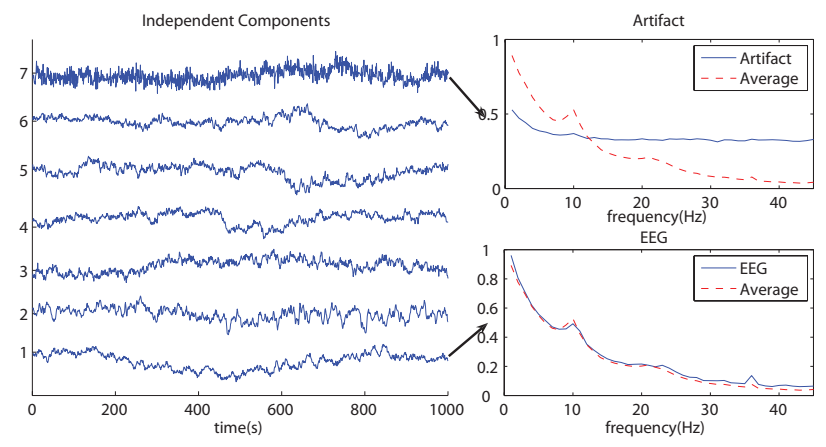

Fig. 2. Segments of ICA results. The left sub figure represents 7 independent components. The right sub figures represent the spectral distribution of EEG and artifact. Here the dashed line represents the average of all the independent component spectral distributions.

\section{B. Spatial Filter}

After performing the preprocessing mentioned above, there still exist a lot of background signals which are unrelated to vigilance estimation. To deal with this problem, we construct a spatial filter based on common spatial patterns (CSP) [11] to extract the EEG signals related to vigilance.

As we know, CSP is only available for labeled two-class problems. However, the task of vigilance estimation is a multi-class problem. To deal with this problem, we use the labeled wakefulness and labeled sleepiness EEG data as the training data to get the common spatial patterns. Then, we choose six common spatial patterns which optimally discriminate between wakefulness and sleepiness as the projection matrix for all of the EEG data. As a result, we have the projected signals $Y$ as follows:

$$
Y=P_{\mathrm{csp}} X
$$

where $X$ denotes the EEG signals, and $P_{\text {csp }}$ denotes the projection matrix.

Since vigilance state transition is a gradually changing process, and the EEG signals from wakefulness to sleepiness change continuously [3], the vigilance-related characteristics of EEG changes are primarily reflected in these projected spatial patterns of wakefulness and sleepiness.

\section{Feature Extraction and Selection}

Here, we use discrete short time Fourier transform to extract spectrum of CSP projected EEG signals as features. The time window is set to 2.56 seconds with 0.56 second overlap. We take the spectra from $1 \mathrm{~Hz}$ to $50 \mathrm{~Hz}$ as the raw feature and use PCA to select the 10 largest eigenvalues, which cover more than $80 \%$ proportion of all of the eigenvalues as the final features.

\section{Clustering Analysis}

Since EEG features are non-stationary during each vigilance state, and vigilance state transition is a gradually changing process, temporal series information is very important for EEG-based vigilance analysis. Almost existing methods, however, don't consider this temporal information. In this paper, we call the existing methods without considering temporal information static approaches, and meanwhile we call the approach considering temporal information dynamic method. Now, we give a brief introduction to the proposed dynamic clustering (DC) algorithm. This method can be regarded as a relational clustering algorithm, which is based on graph factorization and conditional random fields (CRF) [12].

The main idea of the DC algorithm consists of two parts. In the first part, for any pair-wise data relation graph, there exists a latent graph which contains cluster-cluster information and cluster-data information, as depicted in Fig. 3. And the data relation graph is formed from the latent graph. By estimating the latent graph, we can get the datacluster relation, and the cluster-cluster relation. In the second part, considering the gradually changing characteristics of vigilance state transition, any instantaneous vigilance state should be estimated by not only the current EEG observation but also the nearby vigilance states. The structure of the estimation model is shown in Fig. 4.

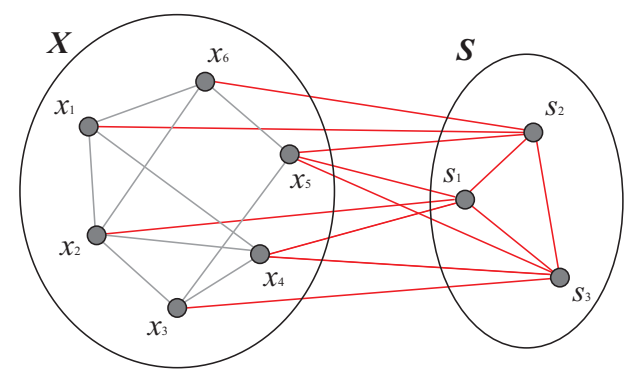

Fig. 3. Pair-wise data relation graph and latent graph. Here $X$ denotes the data set, $S$ denotes the cluster set, and the edges denote the relations among them. The pair-wise data relations graph consists of data set and the corresponding gray line edges. The latent graph consists of the data set, cluster set, and the red edges.

The first phase of the DC algorithm is to construct a graph which can mostly approximate the pair-wise data relation graph. Let $W=\left\{w_{i j}\right\}$ be the affinity matrix of pair-wise data relations, $C=\left\{c_{i j}\right\}$ be the affinity matrix of pair-wise cluster relations, and $B=\left\{b_{i j}\right\}$ be the affinity matrix of 


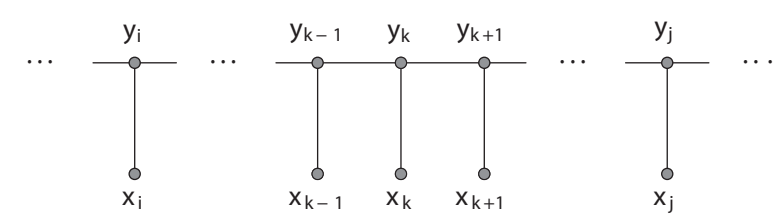

Fig. 4. Structure of the estimation model. Here $x_{i}$ denotes the observation, $y_{i}$ denotes the estimated vigilance state, and the edges denote the relation among them.

relations between data and clusters. From the perspective of Markov random walks, the relations among pair-wise data can be represented by

$$
W=\left(B C B^{T}\right)
$$

where $W$ is known, which can be calculated by the data set, and $C$ and $B$ can be estimated by symmetric convex coding algorithm proposed by [13].

The second phase of the DC algorithm is to find the most probable state sequence by given an observation sequence. Let $\boldsymbol{x}$ and $\boldsymbol{y}$ be the observation sequence and the label sequence (vigilance state sequence), respectively. As the vigilance states are estimated by both the EEG observation and the nearby vigilance states, according to the definition of CRF [12], $(\boldsymbol{x}, \boldsymbol{y})$ is a conditional random field, and the distribution can be formulated as

$$
\begin{aligned}
p(\boldsymbol{y} \mid \boldsymbol{x})= & \propto \exp \left\{\sum_{t} \sum_{i, j \in S} \lambda_{i j} f_{i j}\left(y_{t}, y_{t-1}, x_{t}\right)\right. \\
& \left.+\sum_{t} \sum_{i \in S} \sum_{o \in O} \mu_{i o} g_{i o}\left(y_{t}, y_{t-1}, x_{t}\right)\right\} \\
& f_{i j}\left(y, y^{\prime}, x\right)=1_{y=i} 1_{y^{\prime}=j}, \\
& g_{i o}\left(y, y^{\prime}, x\right)=1_{y=i} 1_{x=o},
\end{aligned}
$$

where $S$ is the set of vigilance states, $O$ is the set of observations, $f_{i j}$ is the feature function for each state pair $(i, j), g_{i o}$ is the feature function for each state-observation pair $(i, o)$, and $\lambda_{i j}$ and $\mu_{i o}$ are the corresponding weights of feature functions. As $\lambda_{i j}$ and $\mu_{i o}$ also reflect the relations among observations and vigilance states, so they can be assigned by $c_{i, j}$ and $b_{i, o}$ obtained by the first phase of the DC algorithm. Then, after all the parameters are determined, the maximum-likelihood label sequence,

$$
\boldsymbol{y}^{*}=\operatorname{argmax}_{\boldsymbol{y}} p(\boldsymbol{y} \mid \boldsymbol{x}),
$$

can be calculated by using Viterbi algorithm. For more detail please refer to [12].

To sum up, the proposed dynamic clustering algorithm can be describe as Algorithm 1.

\section{E. Validation}

After clustering the EEG feature, we use the feedbacks from the subjects, video recordings, and the information from spectrum analysis to validate the clustering results. In spectrum analysis, EEG signals are divided into 5 rhythms, namely $\delta(0.5-3.5 \mathrm{~Hz}), \theta(4-7 \mathrm{~Hz}), \alpha(8-13 \mathrm{~Hz}), \beta(14-25 \mathrm{~Hz})$,

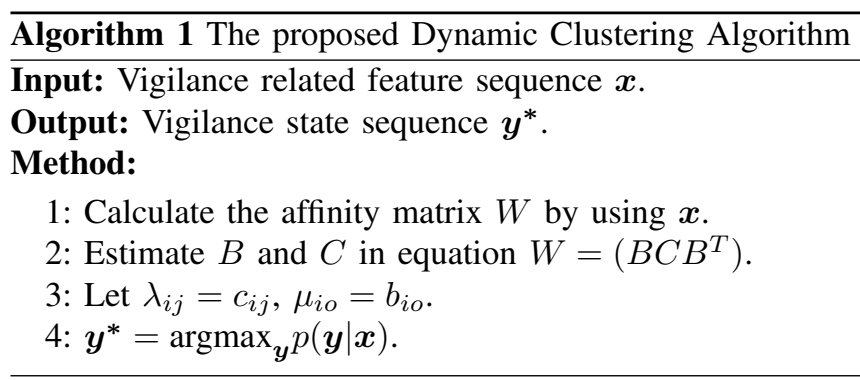

and $\gamma$ (above 26Hz). The spectrum of EEG signals on every rhythm is calculated every one minute. Then, the scalp topography is used to validate the clustering results, which is generated by EEGLAB [14].

\section{RESUlts}

We categorize vigilance levels into four states, namely wakefulness $(W)$, middle state $1\left(M_{1}\right)$, middle state $2\left(M_{2}\right)$, and sleepiness $(S)$. After clustering the EEG signals, the vigilance levels can be ranked using the averaged EEG energy intensity around $\delta$ rhythm in each clustering states. The stronger the EEG energy intensity is, the higher the vigilance level is. In the experiments, the vigilance levels from high to low are $W, M_{1}, M_{2}$ and $S$.

\section{A. Estimation of multiple vigilance states}

We use the DC algorithm to estimate the EEG feature by every 2 seconds. For the 17 subjects, they do not all experience the whole process from wakefulness to sleepiness. Therefore, we choose 7 subjects who experienced the whole process for clustering analysis. Due to the limited space, we only demonstrate one sessions' clustering results, and other sessions have the similar results.

The clustering result of session 12_30 is shown in Fig. 5. This result correctly partitions the labeled wakefulness EEG and the sleepiness EEG. We see that there are some overlaps in this figure. These overlaps do not mean that two states appeared at the same time but because of limited resolution of the figure. During the spectrum analysis, we find that although the EEG spectrum changes gradually, there still exit 4 distinguishable periods, and which match the periods of clustering states harmoniously. Some samples of spectrum analysis result are shown in Fig. 6.

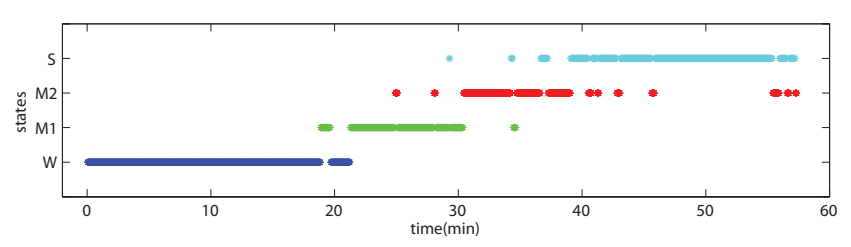

Fig. 5. Clustering result by DC method.

From these spectrum analysis figures we can see that the EEG signals in four clustering states are different. In $\delta$ rhythm, the EEG energy gradually increases from $W$ to $S$. In $\gamma$ rhythm, the EEG energy gradually decreases from 


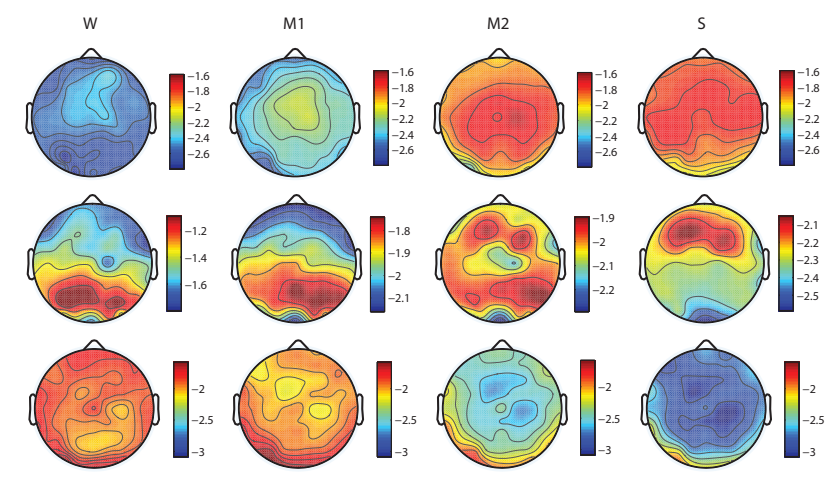

Fig. 6. Scalp topographies of EEG energy proportion in $\delta, \alpha$ and $\gamma$ rhythm averaged on one minute EEG sampled from 4 vigilance states. The top line is the scalp topographies of $\delta$ rhythm, followed by $\alpha$ and $\gamma$ rhythm.

$W$ to $S$. These are all consistent with the former findings. Besides these, we can also find that from $W$ to $S$ the center of EEG energy distribution in $\alpha$ rhythm changes from posterior regions to anterior regions. Usually, $\alpha$ rhythm show its greatest amplitude over posterior regions, and can best be seen during resting periods in which the subjects has his/her eyes closed. And the $\alpha$ rhythm is attenuated when vigilance decreases [15]. And if we look into Fig. 6 carefully, from the color bar, we can find that the EEG energy proportion of $\alpha$ rhythm decreases from wakefulness to sleepiness. So, we think that $\alpha$ rhythm changes from posterior regions to anterior regions is mainly because the attenuation of $\alpha$ rhythm in posterior regions is faster than the attenuation in anterior regions.

\section{B. Performance Comparison}

We use two popular clustering methods, k-means and ncuts, to cluster the EEG signals and compare their performance with that of the DC algorithm. Here, in order to facilitate the performance comparison, we also show the comparison results of session 12_30.
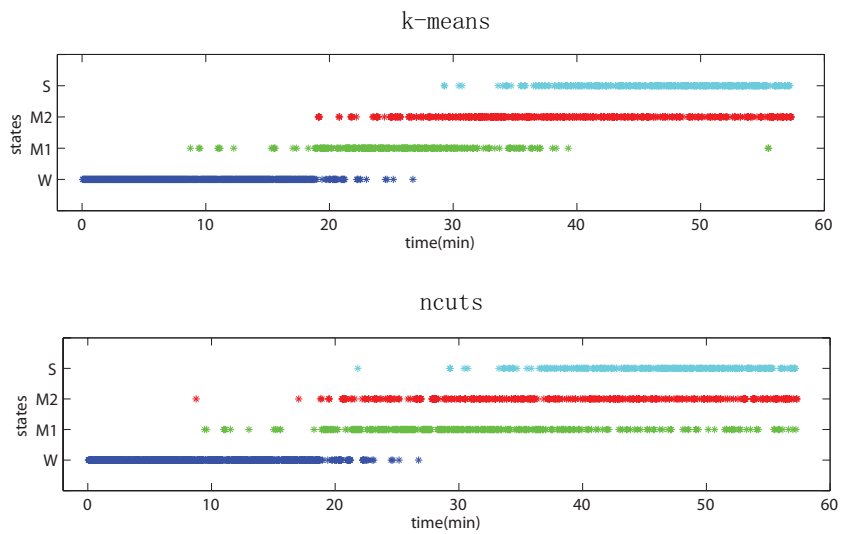

Fig. 7. Clustering results by k-means and ncuts.

Comparing Fig. 5 with Fig. 7, we can see that k-means and ncuts can only distinguish wakefulness from other states. While the DC algorithm can distinguish the four states correctly. The experimental results demonstrate that the proposed DC algorithm is superior to both k-means and ncuts for vigilance clustering analysis.

\section{CONCLUSIONS}

In this paper, we have proposed a dynamic clustering algorithm for vigilance analysis based on EEG signals, which is used for the case that the labeled data are poor and limited. Considering the gradually changing characteristic of vigilance state transition, the proposed dynamic clustering algorithm utilizes the neighborhood information to improve the clustering performance, and can obtain a reasonable grouping of the EEG data at 2 seconds temporal resolution. As future work, we intend to combine the proposed dynamic clustering algorithm with supervised learning method for vigilance analysis.

\section{REFERENCES}

[1] R. Molloy, and R. Parasuraman, "Monitoring an automated system for a single failure: vigilance and task complexity effects", Human Factors, vol. 38, pp. 311-322, 1996.

[2] M. B. Weinger, "Vigilance, Boredom, and Sleepiness", Journal of Clinical Monitoring and Computing, vol. 15, pp. 549-552, 1999.

[3] B. S. Oken, M. C. Salinsky, and S. M. Elsas, "Vigilance, alertness, ro sustained attention: physiological basis and measurement", Clinical Neurophysiology, vol. 117, pp. 1885-1901, 2006.

[4] S. Makeig, T. P. Jung, and T. J. Sejnowski, "Using Feedforward Neural Networks to Monitor Alertness from Changes in EEG Correlation and Coherence", Proceedings of Advances in Neural Information Processing Systems, pp. 931-937, 1996.

[5] T. P. Jung, S. Makeig, M. Stensmo, and T. J. Sejnowski, "Estimating Alertness from the EEG Power Spectrum", IEEE Transactions on Biomedical Engineering, vol. 44, pp. 60-69, 1997.

[6] S. F. Liang, T. C. Lin, R. C. Wu, Y. C. Chen, T. Y. Huang, and T. P. Jung, "Monitoring Driver's Alertness Based on the Driving Performance Estimation and the EEG Power Spectrum Analysis", Proceedings of International Conference of the IEEE Engineering in Medicine and Biology Society, pp. 5738-5741, 2005.

[7] C. Cajochen, J. M. Zeitzer, C. A. Czeisler, and D. J. Dijk, "Doseresponse Relationship for Light Intensity and Ocular and Electroencephalographic Correlates of Human Alertness", Behavioural Brain Research, vol. 115, pp. 75-83, 2000.

[8] L. C. Shi, H. Yu, and B. L. Lu, "Semi-Supervised Clustering for Vigilance Analysis Based on EEG", Proceedinds of International Joint Conference on Neural Networks, pp. 1518-1523, 2007.

[9] J. Shi, and J. Malik, "Normalized Cuts and Image Segmentation", IEEE Transactions on Pattern Analysis and Machine Intelligence, vol. 22, pp. 888-905, 2000.

[10] A. Hyvarinen, J. Karhunen, and E. Oja, Independent Component Analysis, J. Wiley, New York; 2001.

[11] Y. Wang, P. Berg, and M. Scherg, "Common Spatial Subspace Decomposition Applied to Analysis of Brain Responses under Multiple Task Conditions: A Simulation Study", Clinical Neurophysiology, vol. 110, pp. 604-614, 1999.

[12] J. Lafferty, A. Mccallum, and F. Pereira, "Conditional Random Fields: Probabilistic Models for Segmenting and Labeling Sequence Data", Proceedinds of International Conference on Machine Learning, pp. 282-289, 2001.

[13] B. Long, Z. Zhang, X. Xu, and P. Yu, "Relational Clustering by Symmetric Convex Coding", Proceedings of International Conference on Machine Learning, pp. 569-576, 2007.

[14] A. Delorme, and S. Makeig, "EEGLAB: an open source toolbox for analysis of single-trial EEG dynamics including independent component analysis", Journal of Neuroscience Methods, vol. 134, pp. 9-21, 2004.

[15] J. T. Cacioppo, L. G. Tassinary, and G. G. Berntson, Handbook of Psychophysiology Third Edition, Cambridge University, New York; 2007. 